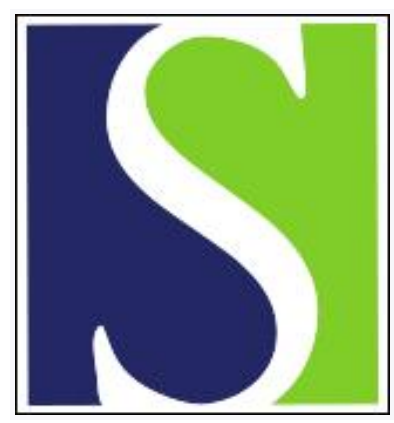

Scand J Work Environ Health 2003;29(4):317-324

https://doi.org/10.5271/sjweh.737

Issue date: Aug 2003

Attenuation of exposure-response curves in occupational cohort studies at high exposure levels

by Stayner L, Steenland K, Dosemeci M, Hertz-Picciotto I

Affiliation: National Institute for Occupational Safety and Health (NIOSH)), 4674 Columbia Parkway, C-15, Cincinnati, OH 45226, United States. LStayner@cdc.gov

Refers to the following texts of the Journal: $1998 ; 24$ suppl $2: 25-41$

$2000 ; 26(1): 37-43$

The following articles refer to this text: $2012 ; 38(5): 418-426$;

2014;40(6):610-620

Key terms: attenuation; discussion paper; epidemiology; exposure-response curve; flat dose-response; high exposure level; occupational cohort study

This article in PubMed: www.ncbi.nlm.nih.gov/pubmed/12934726 


\title{
Attenuation of exposure response curves in occupational cohort studies at high exposure levels
}

\author{
by Leslie Stayner, PhD, ${ }^{1}$ Kyle Steenland, PhD, ${ }^{1}$ Mustafa Dosemeci, PhD, ${ }^{2}$ Irva Hertz-Picciotto, PhD ${ }^{3}$
}

\begin{abstract}
Stayner L, Steenland K, Dosemeci M, Hertz-Picciotto I. Attenuation of exposure-response curves in occupational cohort studies at high exposure levels. Scand J Work Environ Health2003;29(4):317-324.

Numerous occupational cohort mortality studies have observed exposure-response curves to have an increasing slope at low exposure levels that attenuates or even turns negative at high exposure levels. Examples discussed in this paper include dioxin, silica, 1,3-butadiene, cadmium, beryllium, radon daughters, diesel fumes, nickel, arsenic, and hexavalent chromium. Possible explanations for this phenomenon include (i) bias introduced by the healthy worker survivor effect, (ii) a depletion of the number of susceptible people in the population at high exposure levels, (iii) a natural limit on the relative risk for diseases with a high background rate, (iv) mismeasurement or misclassification of exposures, (v) the influence of other risk factors that vary by the level of the main exposure, and (vi) the saturation of key enzyme systems or other processes involved in the development of disease.
\end{abstract}

Key terms epidemiology, flat dose-response.

In our own work with occupational cohort studies, we have observed numerous examples of exposure-response curves, in which the relative risks for chronic diseases tend to attenuate or even decline at high cumulative exposures. At the National Institute for Occupational Safety and Health (NIOSH), we (LS and KS) have observed this phenomenon in 7 of the 10 studies in which we have participated and which included comprehensive exposure-response analyses. These included studies of ethylene oxide and lymphatic and hematopoietic neoplasms (1), dioxin and all cancers (2), silica and kidney disease (3), cadmium (4), beryllium (5), and diesel fumes and lung cancers (6). At the National Cancer Institute (NCI) one of us (MD) has observed this phenomenon in all three of the studies that he has been involved in that had positive exposure-response findings, which included studies of benzene and lymphopoietic neoplasms (7), silica and lung cancer (8), and acrylonitrile and lung cancer (9). One of us (IHP) has previously reported this phenomenon in her work summarizing six studies of arsenic exposure and lung cancer risk, of which four showed a clear flattening at high exposure levels and two were consistent with either a linear or a supralinear exposure-response pattern (10). Other investigators have also reported a similar exposure-response pattern in studies of 1,3-butadiene and leukemia [Delzell E, Sathiakumar N, Macaluso M, Hovinga M, Larson R, Barbone F, et al. A follow-up study of synthetic rubber workers. Unpublished report submitted to The International Institute of Synthetic Rubber Workers, University of Alabama. Birmingham (AL), on 2 October 1995], radon daughters (11), and hexavalent chromium and lung cancer (12). Since relatively few occupational cohort studies have sufficient data to explore the shape of the exposure-response curve, the aforementioned findings are all the more striking.

Log-linear models have been the most commonly used models employed for exposure-response relationships in occupational cohort studies, with the exception

1 Risk Evaluation Branch, Education and Information Division, National Institute for Occupational Safety and Health (NIOSH), Cincinnati, Ohio, United States.

2 Occupational Epidemiology Branch, Division Cancer Epidemiology and Genetics, National Cancer Institute, Bethesda, Maryland, United States.

3 Department of Epidemiology and Prevention, University of California at Davis, Davis, California, United States.

Reprint requests to: Dr Leslie T Stayner, National Institute for Occupational Safety and Health (NIOSH), 4676 Columbia Parkway, C-15, Cincinnati, OH 45226, USA. [E-mail: LStayner@cdc.gov] 
of studies of radiation workers, in which additive linear relative risk models have been frequently used. However, in most of the aforementioned studies, the exposure-response had been found to be represented best by nonlinear relative risk models such as the "power" model (13), which has the following general form:

$$
\mathrm{RR}=[(c+X) / c]^{\beta},
$$

where RR is the rate ratio, $c$ is a constant, $X$ is cumulative exposure, and $\beta$ is the regression coefficient for exposure. The value of $c$ is often fixed at a small number such as 1 , or can be iteratively solved for (14). As an example, we have plotted (figure 1) the results from fitting a power model using conditional logistic regression to the data from a study of dioxin (15).

The phenomenon of a flat or sublinear exposure response has rarely been discussed in the occupational epidemiologic literature. In part, this lack of discussion may be a result of few investigators having examined the shape of the exposure-response relationship in detail in their studies, although this procedure is becoming a more common practice. Hertz-Picciotto \& Smith (10) previously described this phenomenon in several studies of lung cancer and arsenic exposure and discussed the plausibility of several explanations for these findings. The purpose of our present paper is to call further attention to this issue, to elaborate on possible explanations, and to clarify the implications of this phenomenon for epidemiology and risk assessment.

\section{Possible explanations}

\section{Healthy worker survivor effect}

The healthy worker survivor effect refers to the tendency of workers with ill health to drop from the workforce

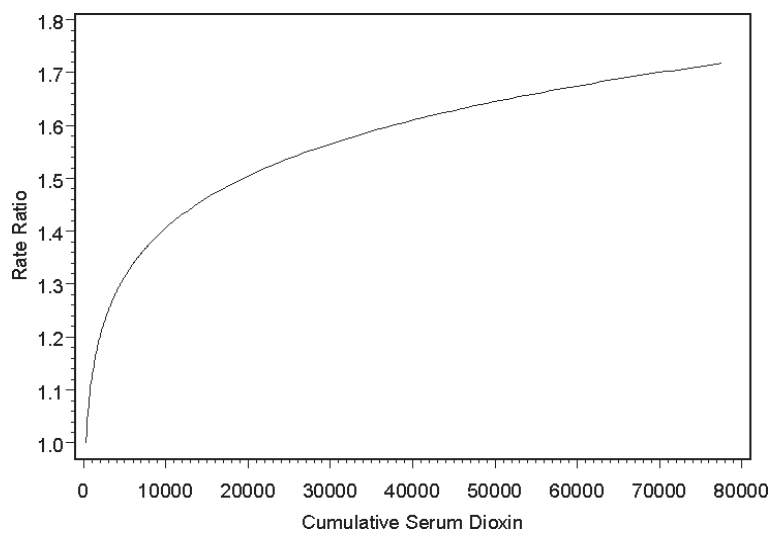

Figure 1. Exposure-response relationship between cumulative serum dioxin level and all cancer mortality observed in the NIOSH study (16). ( $\mathrm{NIOSH}=$ National Institute for Occupational Safety and Health) and the effect this dropout may have on exposure-response relationships in which cumulative exposure is the measure of interest (16). The healthy worker survivor effect is part of the "healthy worker effect" (17). The other part of the healthy worker effect is bias introduced by the initial selection of workers healthy enough to work ("healthy initial hire effect") and the use of general population rates for the comparison group, which includes people who are not healthy enough to work. The bias related to the "healthy initial hire effect" can be avoided by the use of comparisons within the cohort, and such avoidance is generally feasible when exposureresponse relationships are being explored. The healthy worker survivor effect is more difficult to deal with and may introduce bias in exposure-response relationships because short-term workers are often less healthy than long-term workers $(18,19)$ and thus may leave the workplace for reasons either causally or incidentally related to the disease under study. Since short-term workers have higher disease rates than long-term workers, and since, by definition, their exposure is lower, this situation creates a problem of confounding, which could yield negative or flat exposure-response trends when duration or cumulative exposure (product of duration and intensity) is the exposure metric used.

The bias from the healthy worker survivor effect becomes more severe when the exposure itself causes people to leave work because it is noxious (eg, formaldehyde), or because it makes people sick (eg, silica). However, for many chronic diseases with appreciable latency periods and multifactorial origins (eg, cancer), the latter scenario is probably uncommon. Arrighi \& Hertz-Picciotto (20) found that adjustment for the healthy worker survivor effect failed with several methods to change the shape of the exposure-response curve for lung cancer and arsenic exposure, and they concluded that it was unlikely to explain fully the nonlinear exposure-response relationship observed in these studies. In contrast, control for confounding by the healthy worker survivor effect using lagging or controlling for employment status did appear to change the exposure response from a flat to a positive slope in an analysis of cardiovascular disease and arsenic exposure (21).

\section{Depletion of susceptible persons}

If we presume that increasing exposure causes induces higher disease rates in a linear fashion, then there may come a point at which very high exposure levels would be expected to cause disease in virtually everyone in the exposed population. In practice, such a situation is never seen, in part possibly because for most, and possibly all, diseases some sectors of the population are more susceptible than others (eg, due to genetic polymorphisms conferring susceptibility) and there may be a 
depletion of the pool of susceptible people after prolonged exposure. This phenomenon will then give rise to an attenuation of the exposure-response curve at high exposure levels, since the population at risk at high exposure levels contains relatively few susceptible people. As we are generally ignorant of who may be susceptible and who may not, we usually cannot limit our analyses to the susceptible population, for whom perhaps the exposure-response trend might be truly linear.

At higher exposure levels, the relationships between cigarette smoking and both lung and bladder cancer risk have been reported to attenuate $(22,23)$. It has been suggested that this phenomenon may be related to the saturation of the key enzymes involved in the metabolism of components of cigarette smoke, but currently there is little evidence to support this mechanism (22). At least one study provides evidence of susceptible members of the population being depleted. Xu et al (23) found increased lung cancer risk for those having at least one allele of the cytochrome p450 CYP1A1 gene MspI polymorphism. They also observed that the prevalence of this genotype decreased with cumulative exposure to cigarette smoking (pack-years). Thus a larger percentage of people with the high-risk genotypes had low exposures than those with low-risk genotypes; this imbalance, a type of confounding, could explain the flat exposure-response for cigarette smoking and lung cancer.

\section{High background rates of disease}

There is a natural limit to how large the relative risk can be when a disease is common among unexposed people. For example, if a disease had a lifetime risk of $25 \%$ among the unexposed, then the relative risk for an exposed cohort followed for a lifetime could not be greater than 4. Rate ratios are not as limited in this regard as relative risks, since rates of disease or death for any specific disease are theoretically not affected by (ie, are independent of) rates of other diseases. In mortality studies, for example, it is assumed that cause-specific death rates are independent. However, in general, it may be difficult for exposure to result in a very large increase in a cause-specific mortality rate when the cause is common than when it is rare. A high death rate from a specific cause among the exposed population will result in a large number of deaths from this cause at each age considered, and assumptions about the independence of cause-specific rates may not hold under these conditions (24). For example, it may not be possible for exposure to cause a very high rate of cardiovascular disease mortality (a common cause of death) due to exposure in the presence of a high background rate of other causes.

As a counter example, we present, in figure 2, the results from an exposure-response analysis for silica exposure and silicosis (25). In this analysis a power model was also fitted, but the resulting coefficient for cumulative exposure was greater than 1 . This result produces an exposure-response pattern in which the slope of the curve increases with increasing exposure, rather than leveling off with increasing exposure as in the previous examples. The background rate for silicosis among people not occupationally exposed to silica is virtually zero, although a small number of cases might be expected because of possible false-positive X-ray diagnoses. This example illustrates the extent to which rate ratios are free to increase with exposure linearly, sublinearly, or even exponentially for diseases with a zero or low background rate.

A second example is angiosarcoma of the liver and vinyl chloride exposure. Simonato et al (26) have shown a greater than linear increase in rate ratios for angiosarcoma of the liver with increasing cumulative vinyl chloride exposure with rate ratios of 1, 6.8, 24.7, and 45.4 for cumulative exposure of approximately 1000, 4000, 8000, and 15000 (>10000) ppm-years, respectively. This lack of a tailing off of the exposure-response curve has been confirmed by a recent update of follow-up (27), in which the exposure-response coefficient for the bestfitting model using the log of cumulative exposure is greater than 1.0, this finding indicating an upward trend (similar to that in figure 2) at higher exposures for angiosarcoma, a disease which has an extremely low background rate (1 per million).

It is noteworthy that nearly all of the examples (cadmium, arsenic, radon, hexavalent chromium, diesel) identified in this paper are from studies of lung cancer, which has a relatively high background lifetime risk (approximately $5 \%$ ) in the populations studied. One of our examples involved all cancers (dioxin), which has approximately a $25 \%$ lifetime risk. Only two of our examples (butadiene and ethylene oxide) are for relatively rare cancers (lymphopoietic).

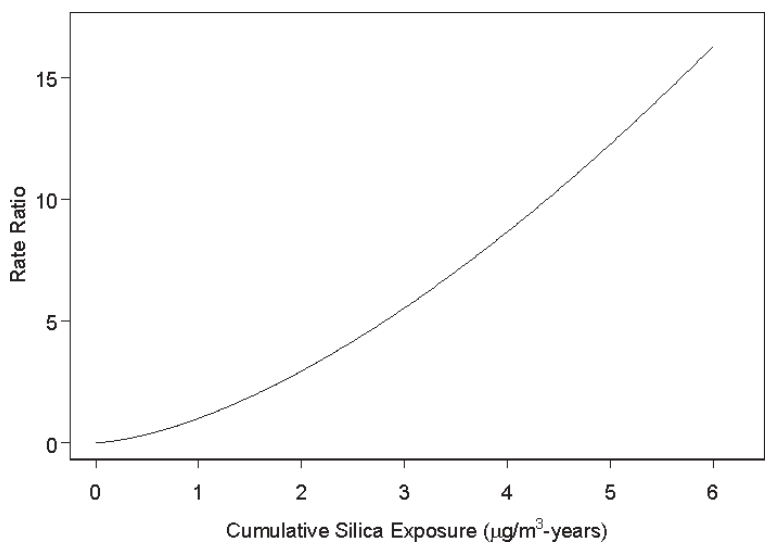

Figure 2. Exposure response relationship between silicosis and cumulative silica exposure in a NIOSH study of gold miners (26). ( $\mathrm{NIOSH}=$ National Institute for Occupational Safety and Health) 


\section{Misclassification or mismeasurement of exposure}

In many common situations, misclassification (for categorical data) or mismeasurement (for continuous data) leads to a biasing of the estimated coefficients of loglinear relative risk models; the direction of this bias is generally towards the null $(28,29)$. However, there are exceptions $(30,31)$ in which trends may be overestimated (bias away from the null). Despite the complexity of the effects of misclassification and measurement error, it is clear that these phenomena can also lead to a flattening of the exposure-response relationship at high exposures for several reasons.

Dosemeci et al (30) has shown how misclassification that is nondifferential with respect to disease status may lead to bias in the slope either away from or towards the null and could even reverse the direction of exposure-response relationships in analyses using polychotomous exposure variables. It is easy to envision how nondifferential exposure misclassification could also result in the flattening of a truly linear exposure-response relationship at high exposure levels. This phenomenon could occur if there is a misclassification error for exposed workers and if there is no error for unexposed workers. This is frequently the case in occupational epidemiology, in which either the general population or an unexposed group of workers is used for reference and is clearly unexposed. This phenomenon may also occur when highly exposed workers are subject to more misclassification than those with lower exposures, which is frequently the case in occupational studies.

To illustrate this potential source of bias, we have performed a simulation in which an unexposed group and five exposed groups were created with 1000 persons in each group. A linear exposure-response relationship was assumed with relative risks of 1.5, 2.0, 3.0, 5.0,

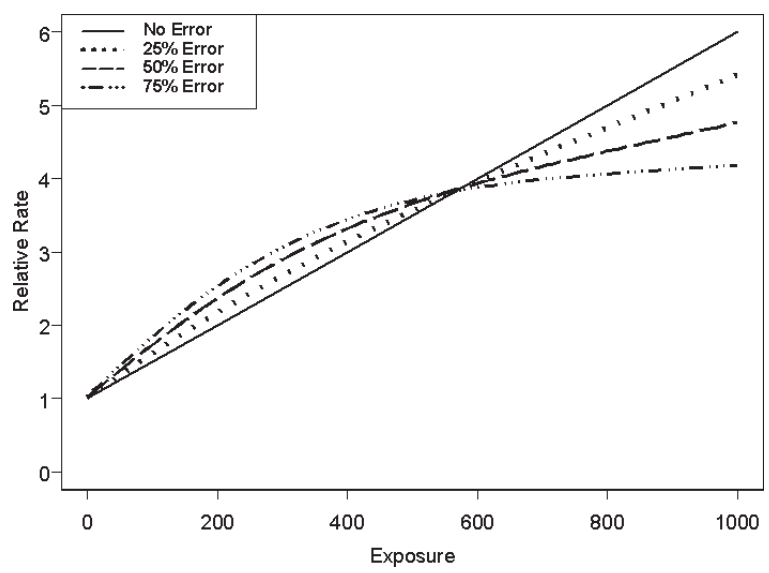

Figure 3. Results from a simulation study of the effect of random misclassification of exposures on a true linear exposure relationship. and 6.0 for the exposed groups $(100,200,400,800$ and 1000 exposure units, respectively). We then introduced varying levels of misclassification error by randomly reassigning a varying percentage (ie, $25 \%, 50 \%$, and $75 \%$ ) of exposed persons to one of the five groups, with equal probability. The unexposed workers were not reassigned, and the exposed persons were not reassigned to the unexposed group. The simulation was repeated 100 times for each of the error levels. The mean results from these simulations are displayed in figure 3. A cubic spline smoother was fitted to the results using SPlus2000 (32) to illustrate the shape of the resulting exposure-response relationships. It can be seen that the exposure-response pattern becomes increasingly nonlinear and demonstrates a flattening of the exposure-response curve at high exposure levels as the proportion of randomly misclassified persons increases.

Systematic errors that lead to either an underestimation of low exposure levels or an overestimation of high exposure levels may also yield an exposure-response curve that will attenuate at higher exposure levels. Inhalation exposure to arsenic and lung cancer risk is an example of how systematic errors in exposure estimates may lead to an attenuation of the exposure-response relationship at high exposure levels. Lubin et al (33), in an analysis of Montana copper smelter workers exposed to arsenic, found that the exposure-response relationship was linear; whereas previous analyses of this cohort had indicated a concave relationship $(10,34)$. The suggested explanation for this discrepancy was that respirator use reduced the inhaled dose for workers whose jobs were in areas of the plant where arsenic exposure was highest (33). Failure to take into account the use of respirators resulted in an overestimation of exposure among highly exposed workers and gave rise to an apparent concave relationship.

Regarding exposure mismeasurement in analyses using continuous exposure variables, even if the error is random, either the attenuation or enhancement of an exposure-response trend may occur. Armstrong (29) has shown that, in a "classical" error measurement situation, when errors are independent of the true exposure, exposure-response relationships are attenuated in relative risk models, in a manner similar to the well-known attenuation in linear regression models. A similar phenomenon can occur with respect to "Berkson" measurement error, although this situation is more complicated and in some situations attenuation does not occur $(28,35,36)$.

Measurement error in some occupational studies can be more severe at high than at low exposure levels; in such cases the attenuation of the slope or flattening of the exposure-response curve would be greater at the high end of the exposure distribution. For example, the highest exposure group in the NIOSH dioxin cohort consisted, 
in large part, of workers who cleaned up spills, and it is known that there was a large degree of variability in the exposure levels of this group. Exclusion of this group from the analysis resulted in a more linear exposure-response relationship than the results for the whole cohort (37).

\section{Influence of other risk factors}

The failure to address the influence of other risk factors adequately in an analysis can lead to distortion of the exposure-response curve from either confounding or effect modification. If a risk factor for the disease under study was more prevalent among people with low exposure than among those with high exposure, then the result could be a flattening of the exposure-response curve at high levels. For example, it may be possible that short-term workers, who would also generally have lower cumulative exposure, are more likely to experience exposures to occupational hazards from other jobs or to be smokers or have other adverse habits than longterm workers do (10). Most occupational studies lack information on employment or exposures experienced outside of the study facility, and many lack information on tobacco consumption.

It is also conceivable that effect modification could produce an attenuation of the slope at high exposure levels. This phenomenon would occur if the effect modification was exposure dependent and was stronger at low exposure levels than at high exposure levels. For example, Hertz-Picciotto et al (38) pointed out that the interaction between smoking and residential (ie, low) exposure to arsenic was multiplicative, whereas the interaction between smoking and occupational (ie, high) exposure was less than multiplicative. In this situation one would expect to observe a stronger slope at low exposure levels than at high exposure levels, even if smoking were nondifferentially distributed with respect to exposure (38).

\section{Saturation of effect}

There are situations in which it is biologically plausible that the response flattens at higher doses because additional exposure intensity is simply biologically irrelevant. Such a situation occurs, in general, when external exposure (exposure intensity) is not proportional to the biologically relevant internal dose. One example is occupational exposure to radon daughters and lung cancer. It has been suggested that the saturation in the exposure-response relationship for lung cancer and radon exposure may be explained by the fact that there are multiple traversals of the cell nuclei by alpha particles at high exposure levels that are "redundant" in that only one traversal is necessary to initiate cancer (39).
Another example is the exposure-response for heart disease and cigarette smoking, which does not increase in a linear fashion with increased intensity of exposure. Law et al (40) have suggested that the lack of a linear increase in risk with increasing exposure may occur because increased platelet aggregation is the principal mechanism causing the excess risk and such aggregation occurs with a very low exposure level, but does not increase appreciably with an increased exposure level. These authors also suggested that the seemingly disproportionate risk of environmental tobacco smoke with respect to heart disease (large effect at a low exposure level) may be due to the same phenomenon.

Finally, in toxicology, there are numerous examples of saturable enzyme systems and their impact on exposure-response relationships (41). A classic example is vinyl chloride, which is metabolically activated to a reactive species by cytochrome P450 enzymes. Figure 4 illustrates the results from two inhalation experiments with rats exposed to vinyl chloride. The amount of radioactive metabolites was quantified following exposure to ${ }^{14}$ carbon-labeled vinyl chloride in one experiment (42), and the incidence of liver angiosarcoma following chronic exposures was assessed in the other (43). Both the tumor response and the metabolism of vinyl chloride appear to attenuate at exposure levels exceeding 500 ppm.

\section{Concluding remarks}

Our primary objective in writing this paper was to call attention to what we believe is a common phenomenon in occupational cohort studies, which is the observation of exposure-response relationships that attenuate at high levels of exposure. This phenomenon was also noted in

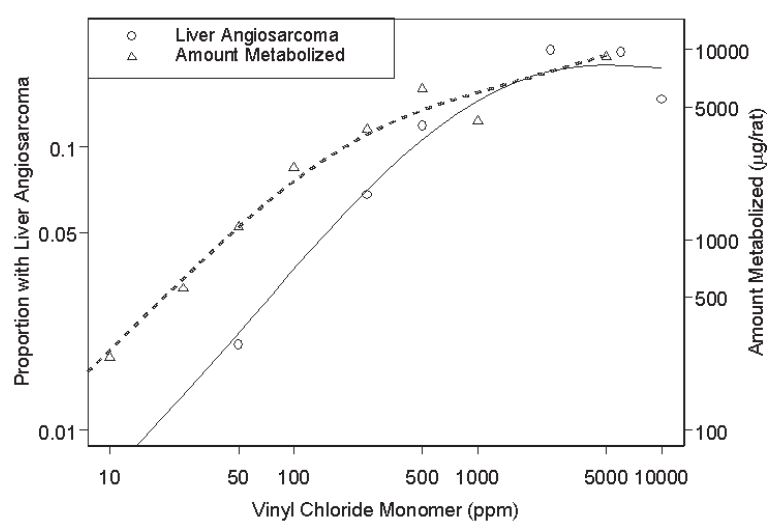

Figure 4. Exposure-response for liver tumors and the metabolism of vinyl chloride in rats. Developed from data presented in studies by Watanabe et al (42) and Maltoni \& Lefemine (43). 
earlier work by Hertz-Picciotto \& Smith for studies of arsenic exposure (10), but our experience suggests that it may be a more common phenomenon than previously recognized. The observation that these exposure-response patterns may commonly occur has important implications for how we analyze and interpret the findings of these studies. For example, a linear exposureresponse relationship for relative risks (rate ratios, odds ratios, standardized mortality ratios) is assumed in a commonly used procedure for testing trends in categorical data (eg, Mantel-Haenzel extension test) (44). The form of the logistic, Cox, and Poisson regression models that most epidemiologists have been using assumes an exponential relationship between exposure and the odds ratio, hazard ratio, or rate ratio and thus that these response measures increase in a more than linear manner with increasing exposure. These tests and models may lead to a false rejection of a positive exposure-response relationship if, in fact, relative risks tend to attenuate at higher exposure levels. Evidence for an exposure-response relationship is one of Bradford-Hill's criteria for supporting causality (45), and it is viewed by many epidemiologists as one of the more critically important criteria. It is also a criterion that often weighs heavily in qualitative assessments of risk, such as the cancer classifications developed by agencies such as the International Agency for Research on Cancer (IARC). It seems particularly inappropriate to reject a causal relationship between exposure and disease altogether because of a lack of a linear exposureresponse trend when a nonlinear relationship may be just as plausible for the reasons discussed in this paper.

Our findings also have important implications for the field of quantitative risk assessment. Many regulatory agencies (eg, the Environmental Protection Agency in the United States) have utilized a linear relative risk model as the default for cancer risk assessments based on epidemiologic data (46). One justification for this use has been based on a multistage theory of cancer (47), which suggests that the dose-response relationship for carcinogens should be linear at low exposure levels. However, the appropriateness of this assumption will depend partly on the shape of the exposure-response relationship, and it would seem inappropriate in a case in which a linear model does not appear to fit the epidemiologic data. In this case it may be preferable to fit a number of parametric models and nonparametric (eg, splines) or more flexible biological models (eg, twostage clonal expansion model) to evaluate potential departures from nonlinearity (14). On the other hand, if the nonlinearity observed in the epidemiologic data is from the healthy worker survivor effect, depletion of susceptible members of the population, or measurement error, then extrapolating to low exposure levels using a nonlinear model may produce biased risk estimates. For example, Stayner et al (4) and Steenland et al (15) chose a linear or piecewise linear model for their cadmium and dioxin risk assessments even though a power model fit the data better because the use of the power model would have resulted in unrealistically high risk estimates at low exposure levels. Of course, extrapolating beyond the range of one's data is always a hazardous task, and, ideally, it should be based upon a biological understanding of the mechanism involved in the disease process whenever possible. Unfortunately our understanding of the disease process has generally been primitive at best, and thus choosing a model for extrapolating beyond the range of epidemiologic data will continue to be a difficult and contentious decision.

Identifying methods to correct the attenuation of exposure-response trends is problematic in part because one rarely knows the reason for the attenuation, but there are some potential solutions. Collecting additional or better information on exposures or covariates may be the only solution for some issues, like the failure to consider the influence of other risk factors. If the attenuation is due to the saturation of a key metabolic pathway or other biological systems, then the attenuation is a real phenomenon, and, in principle, no correction is necessary. However, in this case, improved risk predictions, particularly at low exposures, may be obtained by fitting models using an appropriate biological marker of the target tissue dose, or an estimated dose based on a toxicokinetic model (48), which may linearize the exposure-response relationship. Methods exist for correcting for the healthy worker survivor effect, and they have been applied to correct attenuated or negative exposureresponse relationships with varying success $(20,21)$. Methods also exist for correcting for the mismeasurement or misclassification of exposure (49). These methods have been designed for nutritional studies when a more accurate measurement of exposure ("gold standard") is available for a sample of the study subjects. Unfortunately a "gold standard" for a sample of the workers is rarely available in occupational cohort mortality studies. Finally, an approach that does not require any assumptions about the cause of the attenuation is to simply drop workers with high exposures from the analysis. For example, Park et al (50), in an analysis of silicosis morbidity and mortality, found that the response decreased at high silica exposure levels, and they presented exposure-response models in which the analysis was truncated at a level at which the exposure-response was linear. This approach may be appropriate if one has adequate data at low exposure levels and if one is only interested in the exposure-response relationship at lower levels of exposure.

We wish to emphasize that all of our examples pertain to studies of chronic diseases in which cumulative 
exposure measures were used. It is not clear to what extent this phenomenon may occur in studies of acute effects or in studies that utilize other measures of exposure (eg, average exposure). Some of the factors that we have identified as possible causes of this phenomenon may or not be applicable. For example, the healthy worker survivor effect is unlikely to have an impact on studies that use average exposure, since this factor is generally independent of duration of exposure. On the other hand, in some circumstances, the saturation of an enzymatic pathway might also impact an exposure-response relationship involving an acute effect. Another caveat is that our focus has been restricted to multiplicative models for describing the relationship between exposure and relative risks (eg, rate ratios, odds ratios, or actual relative risks). The phenomena may be model-specific and would not necessarily apply to additive models of absolute rates.

We also note that the phenomenon we describe is not universal and that counter examples can be found in the literature. For instance, at least 11 studies of radon-exposed miners have been conducted, and 9 of these have demonstrated a linear exposure-response relationship (51). The study of Colorado plateau miners (11) that was cited in this paper was the only one to demonstrate clearly statistically significant nonlinearity, although nearly statistically significant $(\mathrm{P}=0.06)$ nonlinearity was observed in the exposure-response relationship of a study of miners in Czechoslovakia, because of a drop of risk at high exposure levels. The fact that an attenuation of the exposure-response relationship has not been consistently observed in the literature for radon may argue against certain explanations (eg, saturation of effect), and for others (eg, bias related to the healthy worker survivor effect or exposure misclassification).

In this paper we have discussed several potential explanations for why exposure-response relationships seem to frequently attenuate with increasing exposure levels in occupational cohort mortality studies. It is probable that there is not just one reason for these findings and that the explanation for these exposure-response effects may vary by compound and study cohort. Other potential explanations not identified in this paper may emerge. It is our hope in writing this paper to raise an awareness of this issue and to generate a discussion of solutions to what we believe is a common phenomenon in occupational epidemiology.

\section{Acknowledgments}

We would like to thank Jay Lubin and Rick Hornung for their help in reviewing earlier versions of this paper and James Bena for his assistance in preparing the graphics for this publication.

\section{References}

1. Stayner L, Steenland K, Greife A, Hornung R, Nowlin S, Morawetz J, et al. An exposure-response analysis of cancer mortality among a cohort of workers exposed to ethylene oxide. Am J Epidemiol 1993;138(10):787-98.

2. Steenland K, Piacitelli L, Deddens J, Fingerhut M, Chang LI. Cancer, heart disease and diabetes in workers exposed to 2,3,7,8-Tetrachlorodibenzo-p-dioxin. J Natl Cancer Inst 1999; 91(9):779-85.

3. Steenland K, Sanderson W, Calvert GW. Kidney disease and arthritis in a cohort study of workers exposed to silica. Epidemiology 2001;12(4):405-12.

4. Stayner L, Smith R, Thun M, Schnorr T, Lemen, R. A doseresponse analysis and quantitative assessment of lung cancer risk and occupational cadmium exposure. Ann Epidemiol 1992;2(3):177-94.

5. Sanderson WT, Ward EM, Steenland K, Petersen MR. Lung cancer case-control study of beryllium workers. Am J Ind Med 2001;39:133-44.

6. Steenland K, Deddens J, Stayner L. Diesel exhaust and lung cancer in the trucking industry exposure-response analysis and risk assessment. Am J Ind Med 1998;34:220-8.

7. Hayes RB, Yin SN, Dosemeci M, Li GL, Wacholder S, Chow WH, et al. Mortality among benzene-exposed workers in China. Environ Health Perspect 1996;10 Suppl 6:1349-52.

8. Cocco P, Rice CH, Chen J-Q, McCawley M, McLaughlin JK, Dosemeci M. Lung cancer risks and silica exposure in Chinese mines and pottery factories: the modifying role of workplace exposure to other lung carcinogens. Am J Ind Med 2001;40: 674-82.

9. Blair A, Stewart PA, Zaebst DD, Pottern L, Zey JN, Bloom TF, et al. Mortality of industrial workers exposed to acrylonitrile. Scand J Work Environ Health 1998;24 Suppl 2:25-41.

10. Hertz-Picciotto I, Smith AH. Observations on the dose-response curve for arsenic exposure and lung cancer. Scand J Work Environ Health 1993;19:217-26.

11. Hornung R, Meinhardt T. Quantitative risk assessment of lung cancer in US uranium miners. Health Phys 1987;52:417-30.

12. Gibb HJ, Lees PSJ, Pinsky PF, Rooney BC. Lung cancer among workers in chromium chemical production. Am J Ind Med 2000;38:115-26.

13. Breslow NE, Day NE. Statistical methods in cancer research; vol II (The design and analysis of cohort studies). Lyon: International Agency for Research on Cancer (IARC); 1987. IARC scientific publication, no 82.

14. Stayner L, Smith R, Bailer J, Luebeck EG, Moolgavkar SH. Modeling epidemiologic studies of occupational cohorts for the quantitative assessment of carcinogenic hazards. Am J Ind Med 1995;27:155-70.

15. Steenland K, Deddens J, Piacitelli L. Risk assessment for 2,3,7,8-p-dioxin (TCDD) based on an epidemiologic study. Am J Epidemiol 2001;154:451-8.

16. Arrighi HM, Hertz-Picciotto I. The evolving concept of the healthy worker survivor effect. Epidemiology 1994;5:189-96.

17. McMichaels MJ. Standardized mortality ratios and the "healthy worker effect": scratching beneath the surface. J Occup Med 1976;18(3):165-81. 
18. Steenland K, Deddens J, Salvan A, Stayner L. Negative bias in exposure-response trends in occupational studies: modeling the healthy worker effect. Am J Epidemiol 1996;143:202-10.

19. Kolstad H, Olsen J. Why do short-term workers have high mortality? Am J Epidemiol 1999;149:347-52.

20. Arrighi HM, Hertz-Picciotto. Controlling the healthy worker survivor effect: an example of arsenic exposure and respiratory cancer. Occup Environ Med 1996;53:455-62.

21. Hertz-Picciotto I, Arrighi HM, Hu S-W. Does arsenic exposure increase the risk for circulatory disease? Am J Epidemiol 2000;151:174-81.

22. Vineis P, Kogevinas M, Simonato L, Brennan P, Boffetta P. Leveling off of the risk of lung and bladder cancer in heavy smokers: an analysis based on multicentric case-control studies and a metabolic interpretation. Mutat Res 2000;463:103-10.

23. Xu X, Kelsey KT, Wiencke JK, Wain JC, Christiani DC. Cytochrome P450 CYP1A1 MspI Polymorphism and lung cancer susceptibility. Cancer Epidemiol Biomarkers Prev 1996;5:687-92.

24. Selvin S. Statistical analysis of epidemiologic data. New York (NY):Oxford University Press; 1996.

25. Steenland K, Deddens J. Increased precision using countermatching in nested case-control studies. Epidemiology 1997; 8:238-42.

26. Simonato L, L'Abbé KA, Andersen A, Belli S, Comba P, Engholm G, et al. A collaborative study of cancer incidence and mortality among vinyl chloride workers. Scand J Work Environ Health 1991;17(3):159-69.

27. Ward E, Boffetta P, Andersen A, Colin D, Comba P, Deddens JA, et al. Update of the follow-up of mortality and cancer incidence among european workers employed in the vinyl chloride industry. Epidemiology 2001;1(6):710-8.

28. Correa-Villasenor A, Stewart W, Franco-Marina F, Hui S. Bias from non-differential misclassification in case-control studies with three exposure levels. Epidemiology 1995;6:276-81.

29. Armstrong B. Effects of measurement errors on relative risk estimates. Am J Epidemiol 1990;132:50-63.

30. osemeci M, Wacholder S, Lubin J. Does nondifferential misclassification of exposure always bias a true effect toward the null value? Am J Epidemiol 1990;132:746-8.

31. Wacholder S. When measurement errors correlate with truth: surprising effects of nondifferential misclassification. Epidemiology 1995;6:157-61.

32. Data Analysis Products Division, MathSoft. S-PLUS 2000 guide to statistics; vol 1. Seattle (WA): MathSoft; 2000.

33. Lubin JH, Pottern LM, Stone BJ, Fraumeni JF. Respiratory cancer in a cohort of copper smelter workers: results from more than 50 years of follow-up. Am J Epidemiol 2000;151(6): 554-65.

34. Lubin JH, Pottern LM, Blot WJ, Tokudome S, Stone BJ, Fraumeni JF. Respiratory cancer among copper smelter workers: recent mortality statistics. J Occup Med 1981;23:779-84.

35. Prentice R. Covariate measurement errors and parameter estimates in a failure time regression model. Biometrika 1982: $69 ; 331-41$.
36. Steenland K, Deddens JA, Zhao S. Biases in estimating the effect of cumulative exposure in log-linear models when estimated exposure levels are assigned. Scan J Work Environ Health 2000;26:37-43.

37. Steenland K, Piacitelli L, Deddens J, Fingerhut M, Chang LI. Cancer, heart disease, and diabetes in workers exposed to 2,3,7,8-tetrachlorodibenzo-p-dioxin. J Natl Cancer Inst 1999; 91(9):779-86.

38. Hertz-Picciotto I, Smith AH, Holtzman D, Lipsett M, Alexeeff G. Synergism between occupational arsenic exposure and smoking in lung cancer induction. Epidemiology 1992;3:23-31.

39. Brenner DJ, Hall EJ, Randers-Pehrson G, Miller RC. Mechanistic considerations on the dose-rate/LET dependence of oncogenic transformation by ionizing radiation. Radiat Res 1993;133:365-9.

40. Law M, Morris J, Wald N. Environmental tobacco smoke exposure and ischaemic heart disease: an evaluation of the evidence. BMJ 1997;315:973-80.

41. Anderson ME. Saturable metabolism and its relationship to toxicity. CRC Crit Rev Toxicol 1981;9(2):105-50.

42. Watanabe PG, McGowan GR, Madrid EO, Gehring PJ. Fate of ${ }^{14} \mathrm{C}$-vinyl chloride following inhalation exposure in rats. Toxicol Appl Pharmacol 1976;37:49-59.

43. Maltoni C, Lefemine G. Carcinogenecity bioassays of vinyl chloride: current results. Ann N Y Acad Sci 1975;246:195.

44. Rothman K, Greenland S. Modern epidemiology. Philadpelphia (PA): Lippincott-Raven; 1998.

45. Bradford Hill. The environment and disease: association or causation. Proc R Soc Med 1965;58:295-300.

46. Environmental Protection Agency (EPA). Guidelines for carcinogen risk assessment. Washington (DC): EPA; 1986. Federal Register, September 24, 33992-34003.

47. Armitage P, Doll R. The age distribution of cancer and a multistage theory of carcinogenesis. Br J Cancer 1954;9(1):112.

48. Kuempel ED, Tran CL, Bailer AJ, Smith RJ, Dankovic DA, Stayner LT. Methodological issues of using observational human data in lung dosimetry models for particulates. Sci Total Environ 2001;274(1-3):67-77.

49. Rosner B, Willet WC, Spiegelman D. Correction of logistic regression relative risk estimates and confidence intervals for systematic within-person measurement error. Stat Med 1989;8(9):1051-69.

50. Park R, Rice F, Stayner L, Smith R, Gilbert S, Checkoway H. Exposure to crystalline silica, silicosis and lung disease other than cancer in diatomaceous earth industry workers: a quantitative risk assessment. Occup Environ Med 2002;59(1):36-43.

51. Lubin JH, Boice JD, Edling C, Hornung RW, Howe G. Radon and lung cancer risk: a joint analysis of 11 underground miners studies. Bethesda (MD): National Institutes of Health, U.S. Dept of Health and Human Services; 1994. Pub. No. NIHHHS-94-3644.

Received for publication: 23 July 2002 